Cinémas

Revue d'études cinématographiques

Journal of Film Studies

\title{
American studies, cultural studies et le cinéma américain des années 80
}

\section{Dana Polan}

Volume 1, numéro 1-2, automne 1990

URI : https://id.erudit.org/iderudit/1000996ar

DOI : https://doi.org/10.7202/1000996ar

Aller au sommaire du numéro

Éditeur(s)

Cinémas

ISSN

1181-6945 (imprimé)

1705-6500 (numérique)

Découvrir la revue

Citer cet article

Polan, D. (1990). American studies, cultural studies et le cinéma américain des années 80. Cinémas, 1(1-2), 132-142. https://doi.org/10.7202/1000996ar

\section{Résumé de l'article}

Abordant le champ des american studies (plus précisément dans son premier essor dans les années 50) et la récente émergence du champ des cultural studies, l'auteur avance que les deux sont incapables de rendre compte de la réelle complexité de la production pour le grand public (dans ce cas, le film américain des années 80), parce qu’ils restent attachés à des notions de culture comme mythe, pourvoyeur de signification, transmetteur de thèmes. L'auteur propose de comprendre la culture contemporaine davantage comme un spectacle - une présentation visuelle saisissante de points de vue non signifiants - et examine au moyen de quelques façons spécifiques comment le film des années 80 travaille à inhiber, à compliquer, à détourner l'interprétation. 


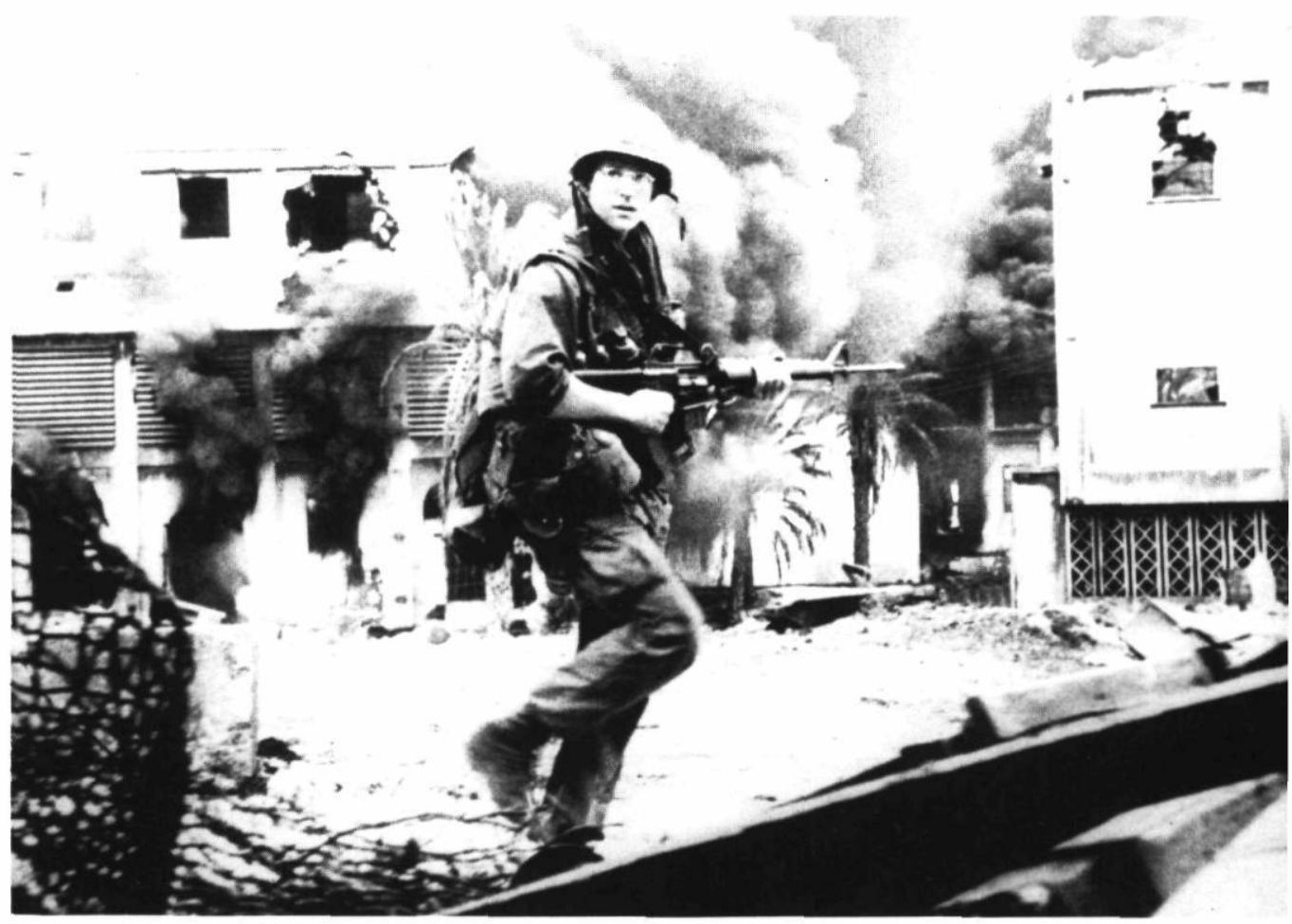

Full Metal Jacket de Stanley Kubrick (1987)

Coll. Cinémathèque québécoise 


\title{
American studies, cultural studies et le cinéma américain des années 80
}

\section{Dana Polan}

\begin{abstract}
RÉSUMÉ
Abordant le champ des american studies (plus précisément dans son premier essor dans les années 50) et la récente émergence du champ des cultural studies, l'auteur avance que les deux sont incapables de rendre compte de la réelle complexité de la production pour le grand public (dans ce cas, le film américain des années 80), parce qu'ils restent attachés à des notions de culture comme mythe, pourvoyeur de signification, transmetteur de thèmes. L'auteur propose de comprendre la culture contemporaine davantage comme un spectacle - une présentation visuelle saisissante de points de vue non signifiants - et examine au moyen de quelques façons spécifiques comment le film des années 80 travaille à inhiber, à compliquer, à détourner l'interprétation.
\end{abstract}

\begin{abstract}
Looking at the field of American Studies (especially in its first flowering in the 1950s) and the newly emergent field of cultural studies, the author argues that both are incapable of dealing with the real complexity of mass cultural production (in this case, American Film in the 1980s) as long as they remain tied to notions of culture as myth, purveyor of meaning, transmitter of theme. The author proposes to understand contemporary culture more as spectacle - the visually gripping presentation of non-signifying sightsand looks at some of the specific ways 1980 s film works to inhibit, complicate, deflect interpretation.
\end{abstract}


Le mouvement que l'on appelle american studies a débuté comme discipline dans les années 50. Contrairement au notoire new criticism, au sein de la critique littéraire, qui s'est éloigné des rapports avec le domaine politique pour se consacrer exclusivement à l'étude formelle, le mouvement american studies a opté pour l'analyse de la représentation des États-Unis. L'Amérique est alors considérée comme un grand texte dont chaque signifiant peut être interprété pour mettre à jour sa valeur mythique. L'œuvre initiatrice est celle de Henry Nash Smith, Virgin Land, publiée en $1950^{1}$. Pour cet auteur, il y a un grand mythe fondateur derrière les activités quotidiennes américaines: la terre comme force à subjuguer, la conquête de l'Ouest comme épreuve à surmonter. D'autres livres de la même époque vont ajouter d'autres mythes à ce grand mythe central: ainsi, le livre de R. W. B. Lewis, American Adam, publié en 1955, aborde l'Américain sous le jour d'un nouveau-né qui jette aux oubliettes les traces européennes de son passé2.

Héritier des american studies ${ }^{3}$, le cultural studies movement a été fondé dans les années 60 au Center for Contemporary Cultural Studies, à Birmingham en Angleterre. Il s'est donné pour but d'analyser comment les groupes culturels spécifiques peuvent refaçonner les messages autour d'eux selon leurs propres besoins et intérêts. Au début, sous l'influence du livre clef The Uses of Literacy, de Richard Hoggart, en 1957, sur la vie quotidienne des ouvriers anglais ${ }^{4}$, le mouvement cultural studies analysait la vie des groupes à l'intérieur du système capitaliste: ainsi le livre célèbre d'Edward Thompson, The Making of the English Working Class, en 1963, étudie la classe ouvrière ${ }^{5}$. Mais c'est surtout avec la publication en 1978 du livre Orientalism, du critique littéraire et homme politique Edward Said ${ }^{6}$, livre sur la constitution par l'Occident de l'Orient, que les cultural studies sont allées au-delà de l'analyse de ce qui se passe à l'intérieur d'une culture pour aborder la question de la confrontation de plusieurs cultures entre elles. L'influence directe de cette approche dans le domaine du cinéma se trouve, par exemple, dans un livre récent d'Ella Shohat, Israeli $\mathrm{Ci}$ nema, directement inspiré par Said7. Shohat examine des formes de pluralité culturelles à la fois à l'intérieur et à l'extérieur d'une société: à l'intérieur, le mélange en Israël des Juifs sépharades, embourgeoisés, et des Juifs askenazes, orientalisés; à l'extérieur, la rencontre entre Juifs et Palestiniens. Cette situation modifie le visage du cinéma israélien qui présentait l'éternelle confrontation de deux mondes irréconciliables, et le présente désormais sous la forme d'un mélange dialogique.

Le mouvement cultural studies rejette avec force l'approche médiatique qui avance que le fonctionnement culturel à l'œuvre est déterminé par le statut social ou politique de ses fabricants et de ses 
récepteurs: c'est le point de vue en grande partie des membres de l'école de Francfort (surtout Adorno et Horkheimer avec leur notion d'industrie de la culture ${ }^{8}$ ). Mais comme l'ont avancé Jacques Rancière et son groupe «Révoltes logiques» dans le livre L'Empire $d u$ sociologue ${ }^{9}$, critique très aiguë de La Distinction de Pierre Bourdieu, une concentration statistique sur l'identité des transmetteurs et des récepteurs des messages fige l'histoire, elle renvoie chaque groupe culturel à une position qu'il doit nécessairement, inévitablement occuper. Pour Bourdieu, chaque groupe social a son goût précis, et il y a des œuvres d'art qui offrent des valeurs figées pour répondre aux besoins propres du groupe. L'œuvre d'art devient un message qui dit toujours la même chose pour son groupe donné.

L'analyse structurale des mythes ${ }^{10}$, bien qu'elle ne se présente pas comme une forme d'analyse médiatique, en adopte les mêmes prémisses: l'art a une signification, cette signification se trouve sous la surface du texte, la découverte de cette signification est en même temps une découverte du fonctionnement culturel du texte.

Dans l'approche cultural studies, on est sûrement entouré par des messages et même par des messages de l'idéologie dite dominante, mais on n'est pas pour autant obligé et déterminé à utiliser ces messages dans leurs sens originaux voulus. L'interprétation culturelle ne peut donc se passer de la sociologie de l'art et doit aussi tenir compte de l'environnement et des fonctionnements divers et potentiellement compliqués ou contradictoires des textes culturels. On peut même avancer l'idée que la culture populaire n'a souvent rien à dire, ne véhicule pas de messages, n'a pas de significations mythiques sous la surface, et que c'est précisément dans son manque de sens qu'elle est idéologique. La conception médiatique de la culture populaire mettant en jeu le réalisme, le narratif et l'illusionnisme pourrait même ne pas correspondre aux textes culturels populaires eux-mêmes.

Mais bien qu'il rejette la notion de culture comme message ou idée déterminante, le mouvement cultural studies reste quand même une analyse axée sur des idées et des contenus. Les chercheurs, dans les cultural studies aujourd'hui, utilisent moins les termes «mythe» et «thème», mais ils restent sur la même voie. On peut noter la propension, chez ces derniers, vers la sémiologie, surtout celle de Roland Barthes dans ses Mythologies ${ }^{11}$, où la fonction d'une culture est de déguiser des connotations tout à fait sociales en dénotations supposées éternelles. Il s'agit donc d'une sémiologie du sens, des significations.

Dans plusieurs études ${ }^{12}$, j'ai essayé de théoriser ce que j'appelle le spectacle, en tentant de nuancer ce terme dans un sens un peu éloigné de son utilisation stéréotypée, surtout dans le domaine du 
cinéma où le spectacle est le gros effet, l'explosion, le grand film de 70 millimètres. Utilisant aussi le mot spectacle dans ce sens stéréotypé, Jean Baudrillard parle du film Syndrome chinois comme non-spectacle dans son refus de nous donner une grande explosion, une fin narrative spectaculaire ${ }^{13}$. L'explosion chaude du cinéma est évitée, dans ce film, au bénéfice d'une démonstration froide ou, comme le dit Baudrillard, cool des pouvoirs des médias dans le monde moderne.

Mais c'est ce $\mathrm{cool}$, cette évacuation des grandes fins narratives que j'essaie dans mes écrits de qualifier de «spectacle». En me concentrant sur le lien étymologique qui renvoie le mot spectacle au mot regard, je pense plutôt que le spectacle est cette force qui détourne la vision narrative et identificatoire d'un film au bénéfice d'une autre vision, celle du film comme suite d'images où le récit ne sert que comme alibi pour faire entrer le spectateur dans un jeu. À l'encontre de l'argument réaliste qui veut que le cinéma populaire doive cacher l'évidence de ses formes sous une histoire racontée dans une sorte de troisième personne, je crois plutôt que le cinéma d'évasion parle souvent de lui-même, rend ses effets et ses formes manifestes, il s'énonce comme à la première personne, présentant un divertissement offert "par quelqu'un», pour les spectateurs. Le spectacle est un art qui ne raconte rien, qui n'est ni réaliste automatiquement, ni auto-réflexif mais qui peut montrer n'importe quoi (y compris son propre dispositif) puisqu'on le regarde pour regarder, pour voir moins une démonstration (soit au sujet du monde, soit au sujet de l'art) que pour voir une monstration, une présentation pure et insensée d'images. La théorie récente du principe mythique, qui regarde les mythes avec un tout autre œil que Claude Lévi-Strauss, permet de préciser ce point de vue. Paul Veyne, dans son livre Les Grecs ont-ils cru à leurs mythes? ${ }^{14}$, avance l'idée qu'il y avait peut-être une croyance, mais pas tant dans un contenu véhiculé par le récit. Ainsi, selon ce dernier, l'auteur grec croit dans la situation immédiate où quelqu'un offre un spectacle verbal, qui est aussi politique et mythique parce qu'il n'y a que certains conteurs qui ont l'autorité de raconter les mythes. Mais le sens du mythe est alors moins important que sa narration: l'effet idéologique du mythe se trouve dans cette narration et non pas dans une idée narrée. De même, ce à quoi l'on croit au cinéma, c'est peut-être le spectacle d'une narration et non pas le contenu de ce spectacle. Bien sûr, le cinéma raconte des histoires, mais elles sont souvent là pour permettre des scènes, des démonstrations visuelles.

En rejetant l'association faite dans la théorie des années 70 entre récit et idéologie dominante, je ne veux pas pour autant refuser de considérer le cinéma populaire comme force sociale, politique ou 
idéologique. Au contraire, je pense que l'on peut trouver ces forces non pas dans les messages, les thèmes, les histoires véhiculées par les films, mais dans l'acte lui-même de présentation d'un spectacle. Comme je l'ai auparavant précisé dans "Cinema and the Ideology of Spectacle", «le monde du spectacle est un monde sans arrière-fond, un monde où les choses n'existent ou ne signifient que par leur «look» (...). Le spectacle offre une image superficielle du monde comme tactique contre un engagement plus critique et plus profond avec ce monde» (Polan, p. 61).

L'image, dans le cinéma contemporain, est souvent gouvernée par une esthétique de la disparition, ce que Marc Vernet appelle «figures de l'absence 15 »: là où la photo pin-up, par exemple, offrait la séduction d'une présence éternelle, l'esthétique de la disparition est celle, pour reprendre les termes de Sartre, d'une présence toujours absente ou toujours en train de s'absenter. Dans un film comme Star Wars (La Guerre des étoiles), où les vaisseaux spatiaux bougent trop vite pour que l'on puisse vraiment saisir les détails, on peut voir dans la disparition un succès de marketing: on ne voit pas tout, donc on y retourne pour voir le film une autre fois. Mais comme le «zappeur» qui imagine qu'il y a toujours quelque chose de mieux sur une autre chaîne, le spectateur dans le cinéma de la disparition est toujours dans une situation désespérée où il ne peut jamais avoir une vision totale du film.

C'est Baudrillard avant tout qui a parlé de la retraite, ou même de la disparition du sens derrière les surfaces de simulation ${ }^{16}$. Dans Evil Demon of Images, il traite spécifiquement du cinéma et suggère comment le cinéma américain évacue le sens pour créer des spectacles purs qui ne disent rien: ainsi Apocalypse Now est un film sans moralité, un film qui, au lieu de commenter la guerre, rivalise avec celle-ci comme spectacle incohérent d'explosions et de jeux visuels. Mais Baudrillard utilise le concept d'hyperréel pour qualifier ce procédé et parle d'un spectateur heureux, pour qui l'Amérique est l'utopie réalisée. Or il y a un autre spectateur, éloigné, rendu distant par le sentiment tragique que le cinéma ne lui donnera pas la plénitude qu'il y cherche - un spectateur qui est poussé par un cynisme et qui s'attend au pire dans toutes les situations.

On peut même supposer que certains rapports avec le cinéma soient masochistes, le spectateur sachant qu'il va être trompé, déçu ou agressé. Ainsi, en voyant des films américains présentés en grandes surfaces, on se rend compte que ce sont ces mêmes grandes surfaces qui sont la cible d'une force destructrice: c'est le cas avec Dawn of the Dead de George Romero ou encore The Blues Brothers Movie. De même, un jeu compliqué de points de vue fait que l'on est successivement dans la position sadique du meurtrier et la 
position masochiste de la victime, dans de nombreux films d'horreur. Dans Blow Out, Brian de Palma utilise aussi cette possibilité de renverser le pouvoir dominateur du spectateur voyeur, de transformer l'ego transcendantal dont parle Metz en moi divisé et masochiste. Le film commence en parodie du film d'horreur avec un travelling du point de vue du meurtrier. À un moment, l'œil-caméra s'approche d'un miroir où l'on aperçoit le visage du meurtrier: un homme bizarre, dont le visage est tordu par des tares affreuses. Puisqu'on a adopté le point de vue de la caméra, cet homme est le spectateur et le miroir renvoie une image perverse, affligeante du voyeurisme et du cinéma.

Plusieurs autres traits semblent caractériser le cinéma américain d'aujourd'hui. Il y a ce qu'on peut qualifier, après Mc Luhan et Baudrillard, de cool - un cinéma qui n'offre pas d'émotions particulières en dehors de celles que l'on éprouve devant n'importe quel spectacle d'images, et ce même dans des films où le sujet semblerait mériter une réponse chaude, engagée. Ainsi, Full Metal Jacket utilise une écriture filmique directe, rigoureuse: tout est là, tout est visible, tout est évident. Or il est impossible de préciser objectivement l'orientation de ce film: on ne sait pas si ce film est contre la guerre ou non. Même une analyse textuelle ne nous permet pas de trancher la question. Contrairement au film Les Bérets verts, de John Wayne, qui utilise un système de champ/contrechamp où chaque objection à la guerre soulevée par le journaliste libéral a sa réponse dans le plan suivant, le champ/contrechamp est assez rare dans Full Metal Jacket, et n'est pas une structure de question-réponse, mais offre dans le visage des acteurs un regard cool, indéchiffrable envers les événements brûlants.

Le cinéma américain traite souvent des grands mythes américains en les vidant de toute émotion. Ici, un film des années 70 , Two-Lane Blacktop (Macadam à deux voies) me semble tout à fait symptomatique de ce point de vue. Deux hommes décident de se lancer dans une course automobile à travers les États-Unis. On se retrouve devant le symbole éternel dont D. H. Lawrence fait l'éloge dans Studies in Classic American Literature 17: la route, le chemin de la découverte, les hommes de la frontière, la rencontre intense entre deux amis ou deux hommes dans un combat amical (comme Rupert et Gerald dans Women in Love). Mais ces mythes sont vidés de sens: les acteurs jouent sans émotion, la course est continuellement interrompue par des détours (amoureux et autres), la caméra reste toujours éloignée de l'action. À la fin, sans émotion, un des deux hommes laisse tomber la course comme si cette décision mettait fin à toute la raison d'être du film, ce dernier se 
terminant avec l'image de la pellicule qui se coince dans le projecteur et brûle.

Une autre façon de déconstruire le mythe du dedans est de réduire le récit à une sorte de squelette, le mythe étant rendu à sa forme tellement pure qu'il devient un objet à regarder passivement, paisiblement: c'est le cas de Coup de cour de Coppola où l'éternelle histoire d'amour n'est qu'un alibi (ou un procédé dans le sens des formalistes russes), une technique pour déclencher un jeu spectaculaire de l'écriture filmique. La minceur de l'intrigue s'oppose alors à la richesse de la cinématographie.

Une autre manière de vider le sens dans les films contemporains est non seulement d'offrir les situations à interpréter ou à évaluer mais aussi d'inclure à la fois des personnages dans l'intrigue qui font eux-mêmes ces interprétations ou ces évaluations. On se trouve dans une situation où tout ce que l'on peut dire et veut dire sur un film est déjà anticipé par le film, incorporé dans son intrigue, annoncé à la surface du film. Il en est ainsi dans Stardust Memories et Les Yeux de Laura Mars.

Mais cette anticipation faite par le film des réponses du spectateur n'est pas obligatoirement dénonciatrice du spectateur. On trouve des films qui flattent l'intelligence du spectateur-intellectuel. Dans une stratégie de marketing qui commence probablement après-guerre et au moment de l'écroulement du système hollywoodien avec son audience homogène, on trouve un cinéma qui sait que sa clientèle est plurielle, composée de plusieurs cultures, y compris une culture intellectuelle. Le cinéma américain devient l'offre de signaux multiples pour des groupes multiples. Pour le cinéphile avisé, un film devient un objet que l'on peut regarder avec distance intellectuelle. Récemment, on a même eu des films qui incorporent des références aux disciplines et aux activités assez spécifiques au monde universitaire (ainsi le film Irreconcilable Differences (Divorce à Hollywood) choisit comme héros un professeur en sémiotique du cinéma (Ryan O'Neil) qui vient de finir un livre sur le cinéma américain des années 40 !). Ces références n'ont pas de sens, ou plutôt elles ne font pas le sens du film: si on est assez avisé pour les remarquer, on se félicite sur sa sagesse style Trivial Pursuit mais on n'a pas pour autant compris un des sens du film.

La tactique d'allusion n'est pas loin d'une autre technique que l'on trouve à l'oeuvre dans le cinéma d'aujourd'hui: ce que j'appelle la figure ou la trope flottante. Une image qui a eu du succès dans un premier film est répétée dans d'autres films, même quand le procédé ne produit pas de sens narratif, afin de créer le sens vague qu'il y a quelque chose de familier. L'exemple type est la salle de bains et la douche dans le film d'horreur, devenus obligatoires depuis Psychose d'Hitchcock. Le film d'horreur devient 
alors moins un genre qui raconte les problèmes des personnages qu'une sorte de mécanisme formel pour enchaîner de nouvelles situations de tuerie, une machine textuelle qui, à partir de certaines prémisses initiales, essaie de voir quelles variations elle peut opérer, un jeu mathématique où l'on expérimente la possibilité de générer de nouvelles actions meurtrières.

Dans le cinéma contemporain, on peut aussi parler de flow ${ }^{18}$. Il y a un flow dans un film lorsque la trame narrative se rompt en scènes souvent contradictoires, souvent incompréhensibles côte à côte, déroulement de scènes où le sens total n'est pas additif mais combatif. Par exemple dans The Burds (Les Banlieues), trois voisins très américains moyens (ou simplement très américains) s'inquiètent de la présence d'une famille d'étrangers d'Europe de l'Est qui s'installe dans le quartier et qui commence à ne pas vivre comme les autres. Soupçonneux, les trois Américains déclenchent catastrophe sur catastrophe en essayant d'entrer dans la maison étrangère pour voir ce qui s'y passe. L'intrusion finale mène à une explosion accidentelle qui détruit la maison et la moitié du quartier et qui manque de tuer le héros américain. En colère, celui-ci se retourne contre ses confrères en criant en gros plans que ce sont eux, les Américains typiques, qui sont les vrais méchants, les vrais étrangers, que c'est l'Américain moyen qui est la menace. Mais, mis dans l'ambulance qui va l'amener à l'hôpital, le héros découvre que les étrangers sont vraiment des meurtriers et qu'ils ont pris la place des vrais médecins. Tout d'un coup, un film qui pendant 90 minutes a condamné le soupçon destructeur défait tout ce qu'il a fait. Mais comme les films de Douglas Sirk, où la fin heureuse ne peut pas faire oublier tout ce qui est arrivé avant la fin, même la fin de The Burds n'est pas le sens du film. Le sens, si sens il y a, se trouve plutôt dans la confusion des deux attitudes et dans le triomphe d'un style filmique qui déclenche des explosions, des scènes d'action, des spectacles purs.

Une autre sorte de flow se trouve entre un film et tout ce qui l'entoure: les pubs, les critiques, mais aussi les messages opposés ou contradictoires dans d'autres films, d'autres récits, d'autres pratiques de la culture. Un bon exemple se trouve dans la thèse de $\mathrm{Ph}$. D. de Barbara Klinger consacrée à Written on the Wind ${ }^{19}$. Klinger montre comment le film n'a pas de sens en lui-même: les sens du film lui sont donnés par les discours publics autour du film. Et cela mène à des contradictions heureuses. Ainsi, la diégèse du film représente le triomphe du foyer, l'idéologie de la vie conjugale domestique, la femme dangereuse comme bouc-émissaire, une idéologie que Fassbinder a qualifiée d'insoutenable. Mais la publicité pour le film représente le contraire: l'amour scandaleux, le 
désir comme la force qui doit franchir toute idéologie de la conjugalité.

Dans plusieurs articles sur la culture postmoderne, le critique américain Frederic Jameson suggère que cette culture est caractérisée par des pratiques de collage, de pastiche, et de schizophrénie ${ }^{20}$. Je suggère que l'on ajoute à cette liste de traits caractéristiques l'incohérence, dans le sens littéral d'une incapacité à trouver une logique, un sens. Est-ce que l'on peut développer des modèles pour rendre compte de cette incohérence et de sa place dans la totalité sociale? Sans une telle élaboration, on risque de regarder un cinéma nouveau avec des méthodes périmées. Ainsi, j'ai été frappé par le décalage entre la complexité de ce qui me semblait être le cinéma moderne (y compris le cinéma populaire moderne) et ce que Deleuze, dans ses deux ouvrages, en disait (du moins dans le peu qu'il en dit: sont absents des livres de Deleuze des cinéastes comme De Palma, Scorsese, Cronenberg, Jarmusch, Joe Dante, etc. ${ }^{21}$. Pour ne prendre qu'un exemple, le Kubrick de Deleuze et le mien sont totalement différents. Exception faite peut-être de 2001, Kubrick offre un cinéma cynique, antihumaniste, où les aspirations de l'esprit et de l'intellect humain sont abaissées par la lourdeur, la «physicalité», l'«instinctualité» du corps humain. Deleuze discute Kubrick comme le point fort d'un cinéma cérébral, un cinéma où la connaissance essaie de s'étaler partout ${ }^{22}$. En harmonie avec son désir de traiter des cinéastes comme penseurs en images, Deleuze construit un Kubrick dont le cinéma est celui des idées: idées comme sujet, idées comme mode d'expression. En revanche, on pourrait ne pas chercher à interpréter Kubrick. L'étonnement de Wendy devant les pages insensées de Jack dans The Shinning peut être notre étonnement devant le cinéma américain moderne: tant de pages, tant d'images, si peu de sens. Wendy ici est le spectateur typique, parfait. Les images sont là, sont claires, mais ce qu'elles signifient, si même elles signifient, est quelque chose que l'on laisse et qui nous laisse dans une indécision cynique...

University of Pittsburgh

\section{NOTES}

1 Henry Nash Smith, Virgin Land: The American West as Symbol and Myth (Cambridge, MA: Harvard University Press, 1950).

2 R. W. B. Lewis, American Adam: Innocence, Tragedy and Tradition in the Nineteenth Century (Chicago: University of Chicago Press, 1955).

3 Voir à ce sujet Patrick Brantlinger, Crusoe's Footprints: Cultural Studies in American and England (New York: Routledge, 1990). 
4 Richard Hoggart, The Uses of Literacy (London: Chatto and Windus, 1957).

5 E. P. Thompson, The Making of the English Working Class (New York: Random House, 1963).

6 Edward Said, Orientalism (New York: Pantheon Books, 1978).

7 Ella Shohat, Israeli Cinema: East/West and the Politics of Representation (Austin, TX: University of Texas Press, 1989).

8 Voir à ce sujet Martin Jay, The Dialectical Imagination: A History of the Frankfurt School and the Institute of Social Research, 1923-1950 (Boston: Little, Brown \& Company, 1973).

9 Voir Collectif «Révoltes logiques», L'Empire du sociologue (Paris: La Découverte, 1984).

10 Voir à ce sujet Claude Lévi-Strauss, «L'Analyse structurale du mythe», Anthropologie structurale (Paris: Plon 1958).

11 Roland Barthes, Mythologies (Paris: Seuil, 1957).

12 Voir, par exemple, "Above all else to make you see: Cinema and the Ideology of Spectacle", Jonathan Arac, éd., Postmodernism and Politics (Minneapolis, MN: University of Minnesota Press, 1986) p. 55-69.

13 Voir Jean Baudrillard, The Evil Demon of Images (Sydney, Australia: Power Publications, 1988).

14 Paul Veyne, Les Grecs ont-ils cru à leurs mythes? (Paris: Seuil, 1983).

15 Marc Vernet, Figures de l'absence (Paris: Éditions de l'Étoile, 1988).

16 Jean Baudrillard, Amérique (Paris: Grasset, 1986) et The Evil Demon of Images, op. cit.

17 D. H. Lawrence, Studies in Classic American Literature, 1923 (New York: Penguin Books, 1977).

18 C'est Raymond Williams qui a introduit le concept dans Television: Technology and Cultural form (New York: Schocken, 1975).

19 Barbara Klinger, Cinema as Social Process, Ph. D, Université d'Iowa, 1986.

20 Voir, surtout, Fredric Jameson, "Postmodernism, or the Cultural Logic of Late Capitalism", New Left Review 146 (1984) p. 53-92.

21 Voir Dana Polan, "Jack and Gilles: Reflections on Deleuze's Cinema of Ideas", Art \& Text 24 (Spring 1989) p. 23-30.

22. Gilles Deleuze, Cinéma 2: l'image-temps (Paris: Éditions de Minuit, 1985) p. 267-68.

\section{OUVRAGE CITÉ}

Polan, Dana. "Above all else to make you see: Cinema and the Ideology of Spectacle". Jonathan Arac, éd. Postmodernism and Politics. Minneapolis, MN: University of Minnesota Press, 1986, p. 55-69. 